\title{
Management als Beziehungsthema
}

\begin{abstract}
Unternehmensführung fokussiert im liberalen ökonomischen Umfeld zumeist auf den Selbstzweck des Unternehmens, statt ihre Versorgungsaufgabe in den Mittelpunkt zu stellen. Beziehungen zum Staat, zur Gesellschaft, zu den Mitarbeitern, ja sogar jene zum Kunden werden nur als Druck wahrgenommen, um die Beziehung zu den Kapitalgebern zu verbessern. Von Robert Mende-Kremnitzer
\end{abstract}

N achhaltiges Management auf der Basis des normativ-kritischen Ansatzes des St. Gallener Management Konzeptes (Rüegg-Stürm/Grand 2015) ist in diesem Blickwinkel jenes, bei dem sich die Beziehung zu keiner Anspruchsgruppe durch die betriebliche Tätigkeit verschlechtert. Dabei sind innerhalb der Beziehung alle unterschiedlichen Interaktionsthemen zu berücksichtigen.

In vielen mikroökonomischen Beziehungen ist der Preis nur ein schlechter Maßstab für die Qualität der Relation. Derselbe Lohn wird vom Unternehmen, an der gewonnenen Produktivität gemessen als tragbar erachtet, während er vom

Mitarbeiter, an seiner Zufriedenheit gemessen, vielleicht als zu gering eingestuft wird. So kann per se nicht gesagt werden, wie viel Produktivität des Unternehmens ein zusätzlicher Glückszustand wert ist.

Eine nachhaltige Beziehung entsteht erst durch eine Balance des Gebens und Nehmens auf beiden Seiten. Anhand des Beispiels der Leistung/Lohn-Beziehung zwischen einem Unternehmen und der Anspruchsgruppe der Mitarbeiter soll das Vier-Seiten-Beziehungsmodell dargestellt werden, das sich auf alle Anspruchsgruppen und Beziehungen erweitern lässt.

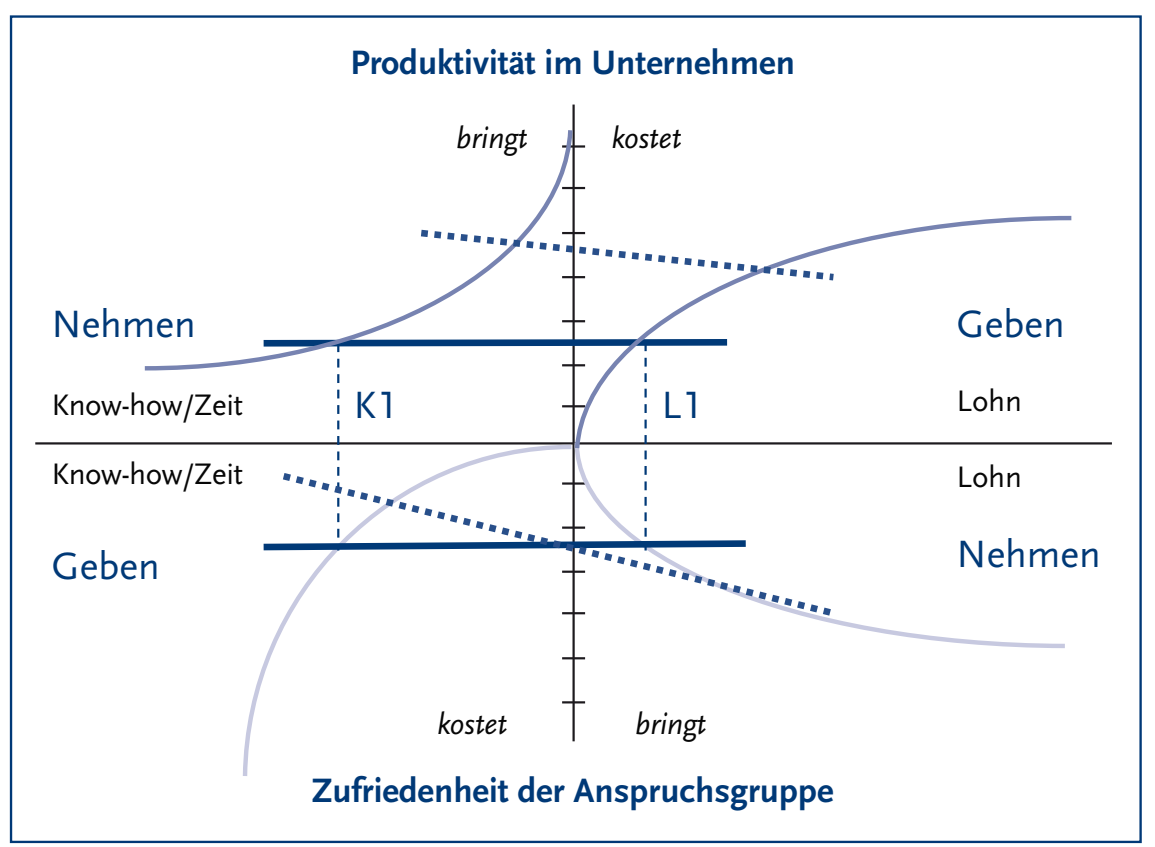

Abbildung 1: Vier-Seiten-Beziehungsmodell der Nachhaltigkeit
Ein Unternehmen nimmt Leistung vom Mitarbeiter in Form von Knowhow/Zeit und misst die Qualität der Beziehung in der gewonnen Produktivität. Die so entstandene Produktivitätsfunktion (linker oberer Quadrant in der Abbildung) zeigt zum Beispiel nach Cobb Douglas abnehmende Grenzwerte. Im Gegenzug gibt das Unternehmen Lohnzahlungen. Jeder zusätzliche Euro wird in vielen Fällen dabei weniger Produktivität bringen (rechter oberer Quadrant).

Auf der anderen Seite bewertet der Mitarbeiter dieselbe Beziehung mit dem Niveau der eigenen Zufriedenheit. Jede zusätzliche Leistung, die er dem Unternehmen gibt, kostet mehr und mehr Zufriedenheit (linker unterer Quadrant). Der dafür erhaltene zusätzliche Lohn bringt jedoch abnehmende Zufriedenheit (rechter unterer Quadrant).

Da jeder Akteur versuchen wird, mehr zu nehmen als zu geben, entstehen einzeln betrachtet schiefe Bilanzstriche (gestrichelte Linien). Zusammengefügt zeigen die vier Bewertungskurven das Ungleichgewicht der subjektiven Bewertungen dieser Beziehung. Nachhaltige Beziehungen kennen jedoch keinen Verlierer bzw. Gewinner. Im Gleichgewichtszustand einer nachhaltigen Beziehung (K1, L1) sind die einzelnen Geben/ Nehmen-Relationen in Balance.

Im nachhaltigen Beziehungsmanagement werden daher alle Themen mit den Stakeholdern in einen Gleichgewichtszustand gebracht. Das betrifft besonders auch die sozialen und ökologischen Themen, die sich von der Steuerzahlung an den Staat bis zur Gleichbehandlung der Mitarbeiter oder der Reduktion der Emissionen gegenüber der Gesellschaft quer durch alle Stakeholder ziehen.

\section{Literatur \\ Rüegg-Stürm, J./Grand, S. (2015): Das St. Gallener Management Modell. Bern, Haupt.}

AUTOR + KONTAKT

Robert Mende-Kremnitzer lehrt seit 14 Jahren Controlling und Nachhaltigkeit an der Fachhochschule Joanneum in Graz, Österreich.

Robert Mende-Kremnitzer, Tel.: +43 6641843520 E-Mail: robert.kremnitzer@fh-joanneum.at 